\title{
Low dose of acetylsalicylic acid and oxidative stress-mediated endothelial dysfunction in diabetes: a short-term evaluation
}

\author{
Eliezer Joseph Tassone $\cdot$ Maria Perticone $\cdot$ Angela Sciacqua $\cdot$ \\ Simona Fortunata Mafrici - Chiara Settino $\cdot$ Natalia Malara • \\ Vincenzo Mollace • Giorgio Sesti · Francesco Perticone
}

Received: 26 May 2014/ Accepted: 2 July 2014/Published online: 6 August 2014

(c) The Author(s) 2014. This article is published with open access at Springerlink.com

\begin{abstract}
Current guidelines suggest the use of low doses of acetylsalicylic acid (ASA) for patients with diabetes mellitus (DM) in primary prevention. However, the evidences demonstrating the beneficial effect of ASA in primary prevention are conflicting. In this pilot study, we evaluated in a group of diabetic patients, in primary prevention, the impact of ASA treatment on oxidative stress and vascular function. We enrolled 22 newly diagnosed diabetic patients, without any previous clinical evidence of cardiovascular disease, to receive, in primary prevention, ASA (100 mg/daily). We tested, in basal condition, after 4 weeks of ASA administration and after 4 weeks of pharmacological washout, the impact of ASA treatment on endothelial function, assessed by
\end{abstract}

Managed by Antonio Secchi.

Eliezer Joseph Tassone and Maria Perticone have contributed equally to this work.

E. J. Tassone - A. Sciacqua - S. F. Mafrici - C. Settino $\cdot$

N. Malara · G. Sesti · F. Perticone $(\square)$

Department of Medical and Surgical Sciences, University Magna

Græcia of Catanzaro, Campus Universitario di Germaneto, V.le

Europa, 88100 Catanzaro, Italy

e-mail: perticone@unicz.it

E. J. Tassone

e-mail: eli.jo@alice.it

A. Sciacqua

e-mail: sciacqua@unicz.it

S. F. Mafrici

e-mail: simonamafrici@hotmail.it

C. Settino

e-mail: chiara.settino@tiscali.it

N. Malara

e-mail: nataliamalara@unicz.it

G. Sesti

e-mail: sesti@unicz.it a semipletysmographic method, measuring the main oxidative stress parameters related to it. As expected, after 4 weeks of treatment, ASA induced a significant reduction of plasma thromboxane- $\mathrm{A}_{2}$, as a consequence of cyclooxygenase- 1 inhibition. By contrast, ASA significantly increased the plasma and urine 8-iso-PGF2 $\alpha$, a well-known prothrombotic molecule, parallel to an increase of plasma NOX2 levels. The enhancement of this oxidative pathway is associated with a significant impairment of endothelial vasodilation, assessed by reactive hyperemia index (RHI). The pharmacological washout reverted all parameters to basal condition. Our findings suggest that ASA utilization for primary prevention in diabetic patients causes a significant increase of oxidative stress burden impairing the vascular function. Present data, if confirmed on a larger population, could permanently discourage the use of the ASA for the primary prevention in patients with DM.

\section{Perticone}

Department of Clinical and Experimental Medicine, University

Magna Græcia of Catanzaro, Campus Universitario di

Germaneto, V.le Europa, 88100 Catanzaro, Italy

e-mail: mariaperticone@ hotmail.com

N. Malara

Interregional Research Center for Food Safety and Health

(IRC-FSH), Catanzaro, Italy

V. Mollace

IRCCS San Raffaele, Rome, Italy

e-mail: mollace@unicz.it

V. Mollace

Department of Health Science, University Magna Græcia of Catanzaro, Campus Universitario di Germaneto, V.le Europa, 88100 Catanzaro, Italy 
Keywords Aspirin - Endothelial dysfunction - Oxidative stress · Diabetes mellitus

\section{Introduction}

Diabetes mellitus (DM) represents one of the most important cardiovascular (CV) risk factors since diabetic patients have an increased risk, two- to fourfold greater, to develop coronary artery disease, myocardial infarction, heart failure, peripheral artery disease, and sudden death [1, 2]. Atherosclerosis is the most important pathogenetic mechanism explaining the relationship between DM and $\mathrm{CV}$ events. It recognizes as the primum movens the endothelial dysfunction, a condition characterized by the reduction of the nitric oxide (NO) bioavailability inducing the impairment of endothelium-mediated vasodilation and the activation of proinflammatory and proliferative pathways.

Hyperglycemia and insulin resistance, present in diabetic disease, induce endothelial dysfunction by several mechanisms, including the advanced glycation end products (AGEs) synthesis, the activation of polyol and hexosamine pathways, and the diabetic dyslipidemia pattern (formation of small and dense low-density lipoprotein (LDL) particles and reduction of high-density lipoprotein cholesterol (HDL-C)), all factors that promote the oxidation/inflammation processes in the vasculature wall [3, 4].

Moreover, a marked increase in oxidative stress, characterized by the overproduction of reactive oxygen species (ROS), has been observed in diabetic patients [5]. The catalytic subunit of the NADPH oxidase (NOX) enzyme NOX2 is the most important cellular source of ROS, which are able to react with various molecules. In particular, the reaction of ROS with arachidonic acid of cell membranes, leads to production of isoprostanes which, in turn, induce platelet aggregation, vasoconstriction and stimulate the inflammatory cascade in the vasculature, all pathogenic mechanisms involved in endothelial dysfunction [6, 7]. The platelet isoprostane 8 -iso-PGF $2 \alpha$ is a vasoconstrictor and platelet activator formed by non-enzymatic oxidation reaction between arachidonic acid and ROS. There are evidences demonstrating that diabetic patients have an upregulation of NOX2 compared to healthy controls, thus presenting higher levels of ROS and 8-iso-PGF2 $\alpha$ [8].

Acetylsalicylic acid (ASA), an inhibitor of cyclooxygenase-1 (COX1) that prevents platelet thromboxane (Tx)$\mathrm{A}_{2}$ formation $[9,10]$, used at low dose (100 mg/daily) in secondary $\mathrm{CV}$ prevention, is able to reduce clinical events in patients with coronary and peripheral artery disease and DM. In fact, platelet $\mathrm{Tx}-\mathrm{A}_{2}$ overproduction, and the subsequent reduction obtained with aspirin administration, has been demonstrated in diabetic patients [11]. Because diabetic patients are characterized by a high CV risk profile, similar to those who have already had a major CV event (myocardial infarction or stroke), it has been proposed the use of ASA for patients with DM also in primary prevention $[12,13]$. The American Diabetes Association, for example, recommends the use of ASA in primary prevention in diabetic patients with 10 -year CV risk $>10 \%$ (men $>50$ years or women aged $>60$ presenting at least one additional major risk factor, such as family history of coronary artery disease, smoking, dyslipidemia, hypertension, or albuminuria) [14]. However, the beneficial effect of ASA in primary prevention is still unclear [15]. In fact, there are recent evidences that attribute to ASA a negative effect on oxidative stressrelated parameters in diabetic patients [16]. Particularly, an oxidative stress-mediated platelet isoprostane overproduction occurs in aspirin-treated diabetic patients that is associated with the attenuation of aspirin-mediated $\mathrm{Tx}-\mathrm{A}_{2}$ inhibition, effect promoting platelet recruitment. Moreover, the NOX2 upregulation observed in diabetic subjects contributes to the ASA-induced formation of 8 -iso-PGF $2 \alpha$ in both platelets and urine [8] that, in turn, increases the burden of oxidative stress.

Although increasing evidences demonstrate a negative impact of ASA on oxidative stress, in primary prevention in diabetic patients, it remains uncertain whether this biological effect is followed, in turn, by functional alterations that contribute to an increased risk of developing atherosclerosis. Because these contrasting evidences, in a recent statement, the FDA expressed an unfavorable opinion to the use of ASA for the primary prevention of heart attack and stroke (http://www.fda.gov/Drugs/ResourcesForYou/ Consumers/ucm390574.htm), by underlying the serious risks of bleeding in the stomach and brain associated with the use of ASA without an effective therapeutic usefulness. Thus, in this study, we evaluated, in a group of newly diagnosed type 2 diabetic patients, the effect of ASA on endothelium-dependent vasodilation and oxidative stress burden, testing the hypothesis that ASA administration (100 $\mathrm{mg} /$ daily) in primary prevention is able to increase the oxidation parameters leading to endothelial dysfunction.

\section{Materials and methods}

\section{Study population}

According to statistical sample size calculation, we enrolled 22 newly diagnosed type 2 diabetic patients, diagnosed by an oral glucose tolerance test (OGTT) [17], without any clinical evidence of $\mathrm{CV}$ diseases. We excluded patients with history of acute vascular events, cardiac arrhythmia or congestive heart failure, type $1 \mathrm{DM}$, chronic kidney disease or serum creatinine level $\geq 1.5 \mathrm{mg} / \mathrm{dL}$, history of cancer, 
infections, smoke, chronic treatment with non-steroidal antiinflammatory drugs, statins, and antiplatelet drugs, in the previous 6 months. At the time of enrollment and during the whole study, all patients were free from any pharmacological treatment. After the enrollment, patients started a 4-week treatment with $100 \mathrm{mg} /$ daily of ASA (T1), followed by a washout period of 4 weeks (T2). The assessment of endothelial function and oxidative stress parameters were performed at baseline and at the end of T1 and T2. Adherence to the treatment was assessed by the pill-count method. The Ethical Committee of University Magna Graecia of Catanzaro approved the protocol, and informed written consent was obtained from all participants. All the investigations were performed in accordance with the principles of the Declaration of Helsinki.

For this study, we did not include the control group for two reasons: at first, because patients were control of themselves; secondly, we preferred not exposing subjects without indications for ASA treatment to a possible bleeding risk.

\section{Laboratory analyses}

\section{Materials}

All materials were from Sigma-Aldrich unless otherwise specified.

\section{Blood sampling}

After a 12-h fast, blood samples were taken. At 08:00 a.m., patients underwent routine biochemical analysis including total cholesterol, HDL cholesterol, LDL cholesterol, triglyceride, glucose, and creatinine. Samples, obtained from patients after supine rest for at least $10 \mathrm{~min}$, were taken in tubes with $3.8 \%$ sodium citrate and centrifuged at $300 \mathrm{rpm}$ for $15 \mathrm{~min}$ to obtain supernatant. Plasma samples were immediately stored at $-80{ }^{\circ} \mathrm{C}$.

\section{$T x-A_{2}$ and NOX2 quantification}

Human Tx- $\mathrm{A}_{2}$ Elisa Kit (Bio-medical assay) and enzymelinked immunosorbent assay kit for cytocrome b-245 $\beta$ polypeptide (cloud-clone corp) were used, respectively, for plasma $\mathrm{Tx}-\mathrm{A}_{2}$ and NOX2 quantitative determinations. Serial dilutions at different concentrations of the standard are prepared. Samples are centrifuged at 1,000 rpm for $10 \mathrm{~min}$, and supernatants are used for the assay. The human $\mathrm{TX}-\mathrm{A}_{2}$ and NOX2 polyclonal antibodies are precoated into 96-well plate. Hundred microliters of standard or samples was added into each well and incubated $90 \mathrm{~min}$ at $37{ }^{\circ} \mathrm{C}$. Plate was washed twice with TBS washing buffer; then, $100 \mu \mathrm{l}$ of working solution, containing biotinylated antihuman $\mathrm{Tx}-\mathrm{A}_{2}$ and antihuman NOX2 detection antibodies (dilution 1:100), was added and incubated at $37{ }^{\circ} \mathrm{C}$ for $60 \mathrm{~min}$. The plate was washed with TBS 3 times and, after $100 \mu$ l Avidin-Biotin-Peroxidase Complex (dilution 1:100) addiction, incubated at $37{ }^{\circ} \mathrm{C}$ for $30 \mathrm{~min}$. The plate was washed 5 times with TBS, and $90 \mu \mathrm{l}$ of TMB color developing agent was added, and then the plate was incubated at $37{ }^{\circ} \mathrm{C}$ for $15 \mathrm{~min}$, in the absence of light. At the end, $100 \mu \mathrm{l}$ of stop solution is added to stop the reaction. The intensity of the color change was measured spectrophotometrically with Victor $^{\mathrm{TM}} \mathrm{X} 4$ multi-label plate reader at the wave length of $450 \mathrm{~nm}$. The standard curve was plotted as the relative optical density (OD) 450 of each standard solution versus the respective concentration of the standard solution. The human Tx- $\mathrm{A}_{2}$ and NOX2 concentration of the samples were interpolated from the standard curve, using curve expert 1.4.

\section{Human 8-isoprostane quantification}

Oxiselect $^{\mathrm{TM}} \quad$ 8-iso-prostaglandin $\mathrm{F} 2 \alpha \quad$ (8-iso-PGF2 $\alpha$ ) ELISA kit (Cell Biolabs, INC.) was used for quantitative determination of human 8-isoprostane in plasma and urines. Hydrolysis of lipoprotein or phospholipid coupled 8 -iso-PGF $2 \alpha$ is required to measure both free and esterified isoprostane. To hydrolyze these ester bounds, plasma was treated with 1 part of $10 \mathrm{~N} \mathrm{NaOH}$ for every 4 parts of liquid samples and incubated for $2 \mathrm{~h}$ at $45^{\circ} \mathrm{C}$. Then, $100 \mu \mathrm{l}$ of $10 \mathrm{~N} \mathrm{HCl}$ per $500 \mu \mathrm{l}$ of hydrolyzed sample is added. These samples were centrifuged for $5 \mathrm{~min}$ at $12,000 \mathrm{rpm}$, and supernatant was used in the assay. Urine samples were acidified to $\mathrm{pH} 3$ by adding $1 / 10$ volume of $1 \mathrm{~N} \mathrm{HCl}$. Different serial dilutions of the standard were prepared. Hundred microliters of diluted antiisoprostane antibody $(1: 1,000)$ was added to the goat antirabbit antibody coated plate and incubated $1 \mathrm{~h}$ at $25^{\circ} \mathrm{C}$ on an orbital shaker. The plate was washed 5 times with wash buffer. Fifty-five microliters of standard or samples and $55 \mu \mathrm{l}$ of 8-isoprostane-HRP conjugate were combined in a microtube, mixing thoroughly. Hundred microliters of the combined solution was transferred per well and incubated $1 \mathrm{~h}$ at $25{ }^{\circ} \mathrm{C}$ on an orbital shaker. The plate was washed 5 times with wash buffer; then $100 \mu$ of substrate solution was added to each well and incubated at room temperature for $20 \mathrm{~min}$ on an orbital shaker. The enzyme reaction was stopped by adding $100 \mu$ l of stop solution to each well. Immediately, we read absorbance of each well on Victor ${ }^{\mathrm{TM}}$ X4 multi-label plate reader at the wave length of $450 \mathrm{~nm}$. The standard curve was plotted as the relative (OD) $450 \mathrm{~mm}$ of each standard solution versus the respective concentration of the standard solution. The human 8-isoprostane concentration of the samples was interpolated from the standard curve. 


\section{Endothelial function}

Endothelial function was evaluated by a semipletysmographic method, with the measurement of digital pulse volume amplitude (PVA). PVA was measured with the subjects placed in the supine position, in a quiet, temperature-controlled environment set at $22{ }^{\circ} \mathrm{C}$. A peripheral arterial tonometer (PAT) was used to measure PVA in the fingertip of the index finger (Itamar-Medical, Caesarea, Israel). The peripheral arterial tonometer apparatus consists of a finger-mounted probe that surrounds the fingertip with an electronically controlled, inflatable, pressurized air cushion confined within a rigid external case. The pressure changes within the probe that accompany PVA changes in the fingertip is transmitted to a personal computer where the signal is band-pass filtered $(0.3-30 \mathrm{~Hz})$, amplified, displayed, and stored. PVA was analyzed at rest and during reactive hyperemia $(\mathrm{RH})$. $\mathrm{RH}$ was elicited by the release of an upper arm blood pressure cuff inflated above systolic pressure for 5 min. Digital PVA-RH was calculated as the ratio of the average PVA over a one-minute time interval starting 1 min after cuff deflation $(\mathrm{RH})$ divided by the average PVA measured for $1 \mathrm{~min}$ before cuff inflation (baseline) (RH index, RHI). The PVA from the index finger of the other, non-ischemic hand (which was not subject to $\mathrm{RH}$ ), was measured continuously throughout the study to assess any drift in the magnitude of the signal due to systemic factors.

\section{Statistical analysis}

We computed the minimum sample size with respect to a two-tailed one-sample Student's $t$ test, considering: a difference for RHI variation to be detected between baseline and after ASA treatment, $|\mathrm{d}| \geq 15 \%$; standard deviation of the paired differences, $\mathrm{SD}=0,11$; and type 1 error probability $\alpha=0.05$ and power $1 \_b=0.90(0.80)$. Differences among different times for the oxidative stress parameters, (in particular, urinary and plasma 8-iso-PGF2 $\alpha$, plasma Tx$\mathrm{A}_{2}$ and NOX2), and vascular function were evaluated by ANOVA for repeated measures. Chi-squared test will be utilized for categorical variables. Data are reported as mean \pm SD. Differences were assumed to be significant at $P<0.05$. All comparisons were performed using the Statistical Package of Social Science (SPSS) version 16.0 for Windows (SPSS Inc., Chicago, IL, USA).

\section{Results}

\section{Study population}

In Table 1, we reported the anthropometric, biochemical, and hemodynamic characteristics of the study population, expressed as mean $\pm \mathrm{SD}$. As evident, the group consists of 11 men and 11 women (mean age $59.6 \pm 6.5$ years) with mean blood pressure values of $134.7 / 83.4 \mathrm{mmHg}$. Moreover, lipid profile showed mild hypercholesterolemia and hypertriglyceridemia, consistent with their clinical condition of DM. No significant differences were observed at the end of study (Table 1).

Effects of ASA treatment on Tx- $\mathrm{A}_{2}$ and 8-iso-PGF2 $\alpha$ levels

The antiplatelet effect of ASA, evaluated by plasma Tx- $\mathrm{A}_{2}$ measurement, was observed after 4 weeks of treatment (from $8.25 \pm 1.45$ to $6.95 \pm 1.08 \mathrm{pg} / \mathrm{ml}, P=0.013$ ). At
Table 1 Anthropometric, biochemical, and hemodynamic characteristics of the study population in basal
$B M I$ body mass index, $S B P$ systolic blood pressure, $D B P$ diastolic blood pressure, $P P$ pulse pressure

\begin{tabular}{llll}
\hline & Basal & End of study & $P$ \\
\hline Gender $(\mathrm{m} / \mathrm{f})$ & $11 / 11$ & $11 / 11$ & - \\
Age $($ years $)$ & $59.6 \pm 6.5$ & $59.6 \pm 6.5$ & - \\
BMI $\left(\mathrm{kg} / \mathrm{m}^{2}\right)$ & $29.3 \pm 2.6$ & $29.0 \pm 3.1$ & 0.808 \\
SBP $(\mathrm{mmHg})$ & $134.7 \pm 11.0$ & $130.5 \pm 14.7$ & 0.457 \\
DBP $(\mathrm{mmHg})$ & $83.4 \pm 9.0$ & $81.0 \pm 11.4$ & 0.590 \\
PP $(\mathrm{mmHg})$ & $51.3 \pm 9.7$ & $49.5 \pm 12.8$ & 0.714 \\
Total cholesterol (mg/dl) & $208.8 \pm 22.5$ & $202.3 \pm 17.7$ & 0.460 \\
HDL cholesterol (mg/dl) & $42.7 \pm 10.7$ & $38.7 \pm 10.3$ & 0.382 \\
LDL cholesterol $(\mathrm{mg} / \mathrm{dl})$ & $139.2 \pm 23.7$ & $141.8 \pm 26.5$ & 0.811 \\
Triglyceride $(\mathrm{mg} / \mathrm{dl})$ & $134.5 \pm 36.8$ & $108.5 \pm 42.7$ & 0.142 \\
Fasting glucose $(\mathrm{mg} / \mathrm{dl})$ & $124.0 \pm 38.0$ & $122.4 \pm 24.2$ & 0.907 \\
Fasting insulin $(\mathrm{mU} / \mathrm{ml})$ & $20.9 \pm 6.6$ & $19.3 \pm 4.9$ & 0.526 \\
HOMA index & $6.0 \pm 1.7$ & $5.9 \pm 1.4$ & 0.882 \\
HbA1c $(\% ; \mathrm{mmol} / \mathrm{mol})$ & $6.5 \pm 0.9 ; 48.0 \pm 2.7$ & $6.4 \pm 1.2 ; 46.0 \pm 3.6$ & 0.827 \\
Creatinine $(\mathrm{mg} / \mathrm{dl})$ & $0.9 \pm 0.4$ & $0.8 \pm 0.7$ & 0.685 \\
\hline
\end{tabular}




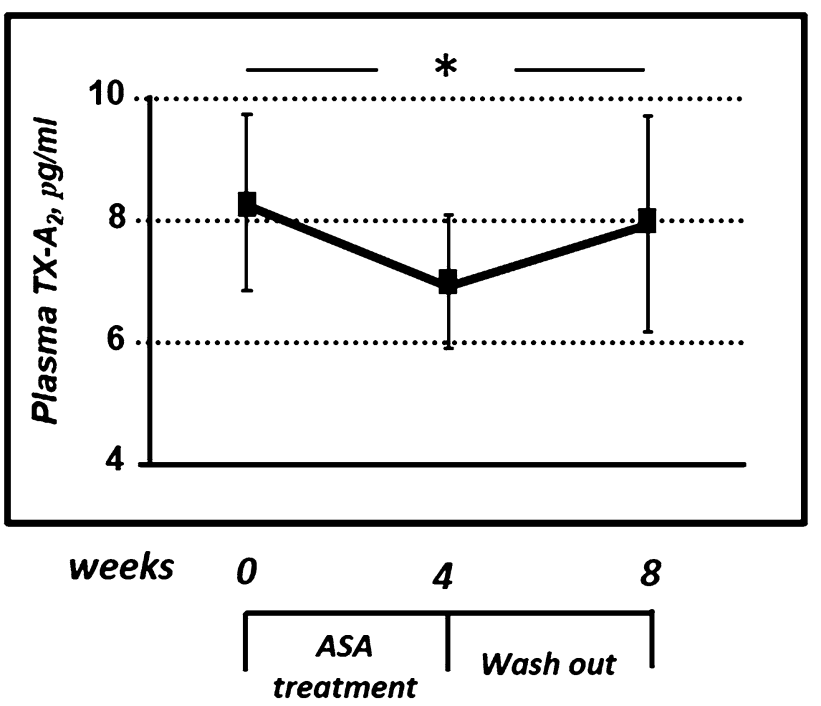

Fig. 1 Effects of ASA on plasma levels of Tx-A $\mathrm{A}_{2}$ in diabetic patients. ASA treatment induced a significant reduction of $\mathrm{Tx}-\mathrm{A}_{2}$ levels, as consequence of COX1 inhibition. $*=P<0.05$ by ANOVA for repeated measures

the end of pharmacological washout, Tx- $\mathrm{A}_{2}$ levels significantly increased, reaching almost basal values (Fig. 1). As observed in Fig. 2, treatment with ASA $100 \mathrm{mg} /$ day induced a significant increase in plasma (from $173.0 \pm 13.5$ to $187.5 \pm 14.8 \mu \mathrm{g} / \mathrm{ml}, \quad P=0.009)$ and urine $(79.5 \pm 17.5$ to $107.0 \pm 15.5 \mu \mathrm{g} / \mathrm{ml}, P<0.0001)$ 8 -iso-PGF2 $\alpha$, respectively. Discontinuation of treatment for 4 weeks (washout) resulted in a significant reduction in 8 -iso-PGF2 $\alpha$ levels, similar to that observed in baseline conditions (Fig. 2).

Effects of ASA treatment on NOX2 levels

Four weeks administration of ASA was able to induce a NOX2 overexpression. In particular, NOX2 levels increased in plasma samples, from $7.93 \pm 0.57$ to $8.75 \pm 1.38 \mathrm{ng} / \mathrm{ml}(P=0.010)$. Interestingly, after the washout period, NOX2 levels decreased, returning nearly to basal levels (Fig. 3).

Effects of ASA treatment on endothelial function

Effects of ASA treatment on vascular function, expressed by RHI, are shown in Fig. 4. As observed, the administration of $100 \mathrm{mg} /$ day of ASA induced, already after 1 week, a significant reduction of the vasodilatory response to ischemia that reached the maximal reduction after 4 weeks of treatment (RHI from $1.72 \pm 0.30$ to $1.33 \pm 0.26, \quad P<0.0001)$; normal value $\geq 1.67$. After 4 weeks of pharmacological washout, endothelial function was completely restored, with average values of RHI
A

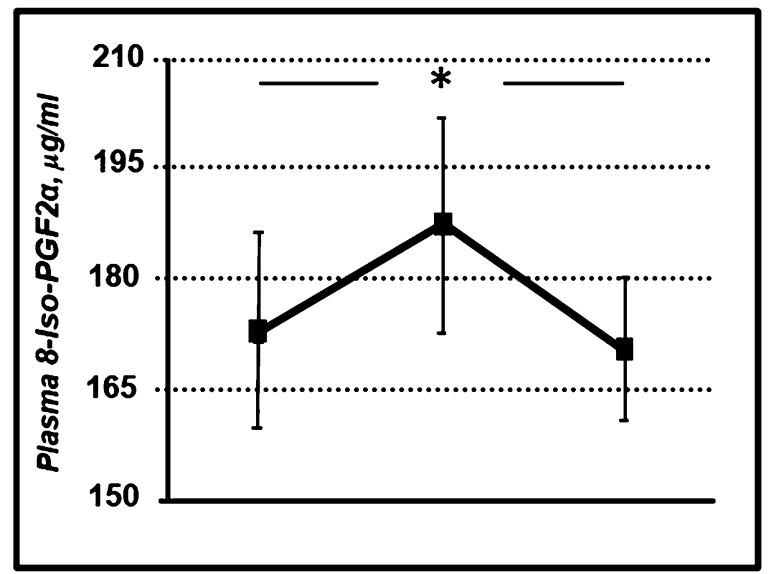

B

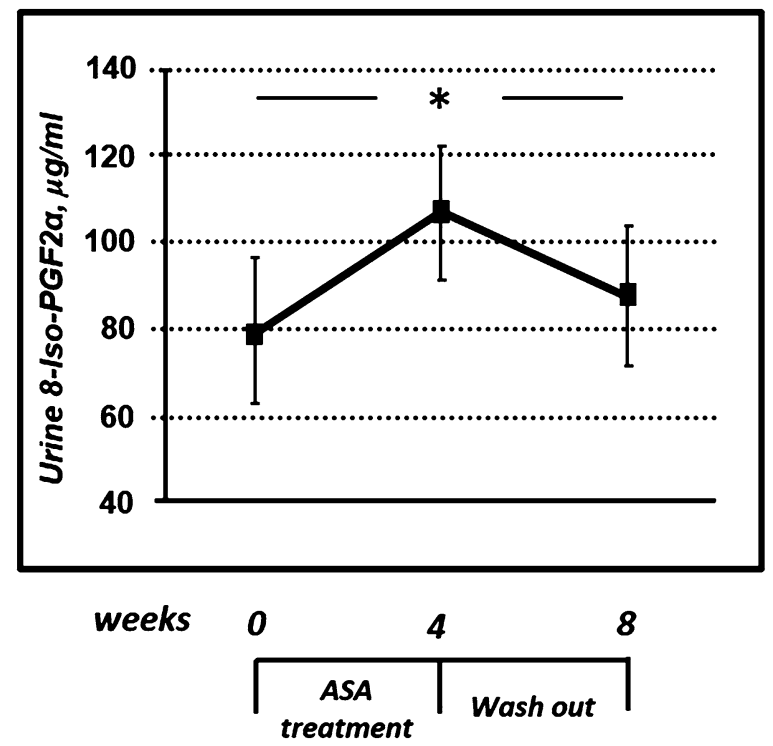

Fig. 2 Effects of ASA on urinary a and plasma b levels of 8-isoPGF $2 \alpha$ in diabetic patients. ASA treatment was able to induce a significant increase of 8 -iso-PGF2 $\alpha$ levels with a reduction after 4 weeks of drug washout. $*=P<0.05$ by ANOVA for repeated measures

similar to that observed in basal conditions $(\mathrm{RHI}=1.75 \pm 0.17)$.

\section{Discussion}

In this study, we evaluated, in a group of newly diagnosed type 2 diabetic patients, the impact of ASA treatment in primary prevention on oxidative stress and vascular function. We demonstrated that ASA administration induces a significant increase in global oxidative burden, as shown by the increase of plasma and urine 8-iso-PGF2 $\alpha$, a wellknown proaggregating and prothrombotic molecule [18]. As already speculated, the increase of 8-iso-PGF2 $\alpha$ could 


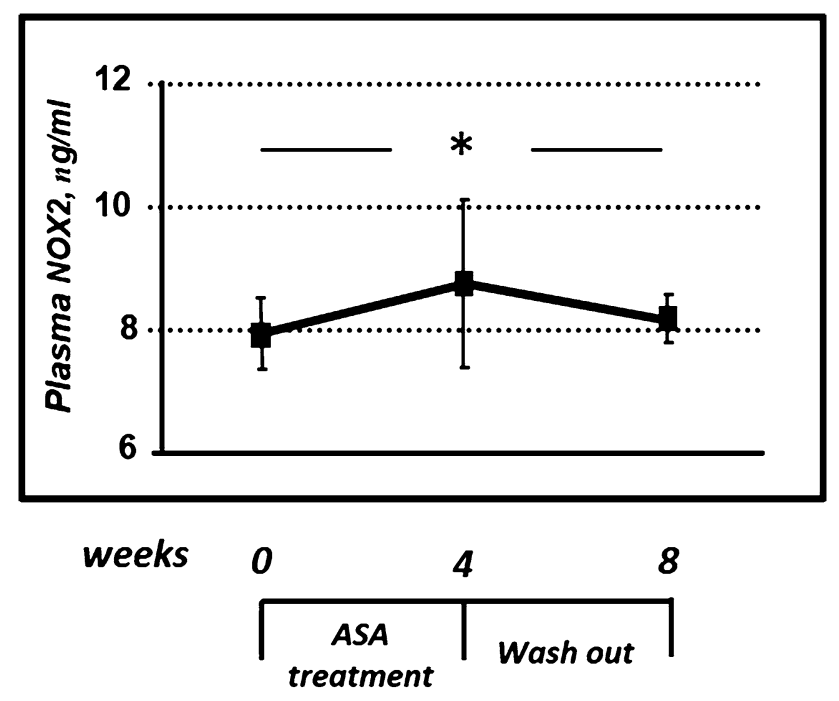

Fig. 3 Effects of ASA on NOX2 plasma levels in diabetic patients. ASA treatment was able to induce a significant increase of NOX2 levels, which returned to basal values after 4 weeks of pharmacological washout. $*=P<0.05$ by ANOVA for repeated measures

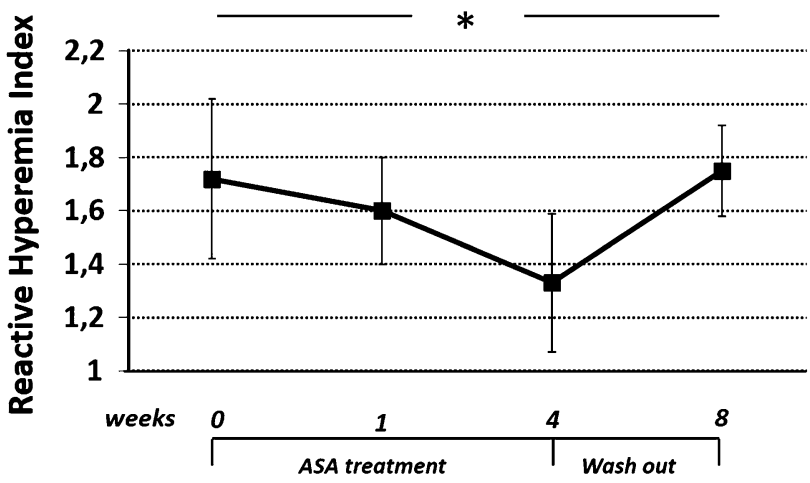

Fig. 4 Effects of ASA on endothelium-dependent vasodilation assessed by reactive hyperemic index (RHI). ASA treatment in diabetic subjects caused a significant reduction of endothelial vasodilatory response to ischemia; after the washout period, RHI values were similar to those observed in basal condition. $P<0.0001$ by ANOVA for repeated measures

be promoted, indirectly, by the ASA-induced inhibition of the COX1 enzyme that, in turn, leads to the triggering of an escape pathway of isoprostanes formation [16, 19]. Moreover, in diabetic patients, there is per se an increased activity of the NADPH oxidase that is responsible for the increased production of ROS [8], which are able to interact with arachidonic acid to form isoprostanes [20]. ASA treatment is also able to increase NOX2 levels, contributing to cellular oxidative burden in ROS production. In this way, the treatment with ASA, by selectively inhibiting COX1, prevents the arachidonic acid contribution to generate $\mathrm{Tx}-\mathrm{A}_{2}$, favoring the switch of the substrate for the isoprostanes production [21]. According to this, our data show that ASA induces a significant reduction in plasma Tx- $\mathrm{A}_{2}$ and increases the plasma and urinary levels of 8-isoPGF2 $\alpha$ (Fig. 5). Of interest, Gonçalves and corkers recently reported that concomitant use of metformin and ASA in diabetic patients can reduce levels of urinary biomarkers of oxidative stress [22], offering new important therapeutic implications for antidiabetic drugs.

The most relevant data of our study are the demonstration that ASA treatment in type 2 diabetic patients causes a significant reduction in the endothelium-dependent vasodilatory response. The reduction of RHI, already occurred after 1 week of treatment, which reached pathological values after 4 weeks, demonstrates that the ASAincreased oxidative stress could adversely affect the vascular function, by inhibiting its vasodilatory properties in a setting of patients considered at high CV risk also in primary prevention. This finding has an important clinical implication, because endothelial dysfunction represents a powerful negative prognostic factor in different setting of patients, including the uncomplicated hypertensive patients that are considered at low risk in comparison with diabetic ones [23]. Moreover, the impairment of endothelial function might worse the metabolic control since endothelial dysfunction interacts with some different molecular pathways involved in the appearance of new diabetes [3, 24].

These results are clinically relevant because, according to current evidences of the literature, there is still no unanimous opinion about the real need of ASA treatment for the primary cardiovascular prevention [25]. Our data show that in DM, in which oxidative stress plays a pivotal role in the pathogenesis of vascular damage, the treatment with aspirin may increase the oxidative stress burden, worsening in turn the vascular function. This negative effect of ASA could have an important impact, especially in diabetic patients who undergo percutaneous coronary angioplasty and stenting, for whom the association of ASA with another antiplatelet agent is recommended. This treatment, on the basis of our findings, could be potentially dangerous because it reduces the NO bioavailability with a consequent worsening of vascular function. Moreover, our results contribute to clarify an important pathophysiological mechanism with a relevant clinical application because they consent to better interpret the controversial results obtained in primary prevention of diabetic patients [15]. This is further supported by the fact that ASA, beyond the observed negative impact on vascular function, increases the bleeding risk, mainly in subjects without previous $\mathrm{CV}$ events in which a clear benefit of ASA utilization is not clearly demonstrated. Our results differ from those reported by Raghavan et al. [26], but these differences are due to the methods and study population characteristics. In fact, these Authors evaluated diabetic patients treated with statins, 
Fig. 5 Diagram graphically reports the effects of ASA on vascular oxidative stress leading to endothelial dysfunction. COX1 inhibition triggers an escape pathway that is able to promote the production of isoprostanes that, in turn, contribute to impair the endothelium-dependent vasodilation

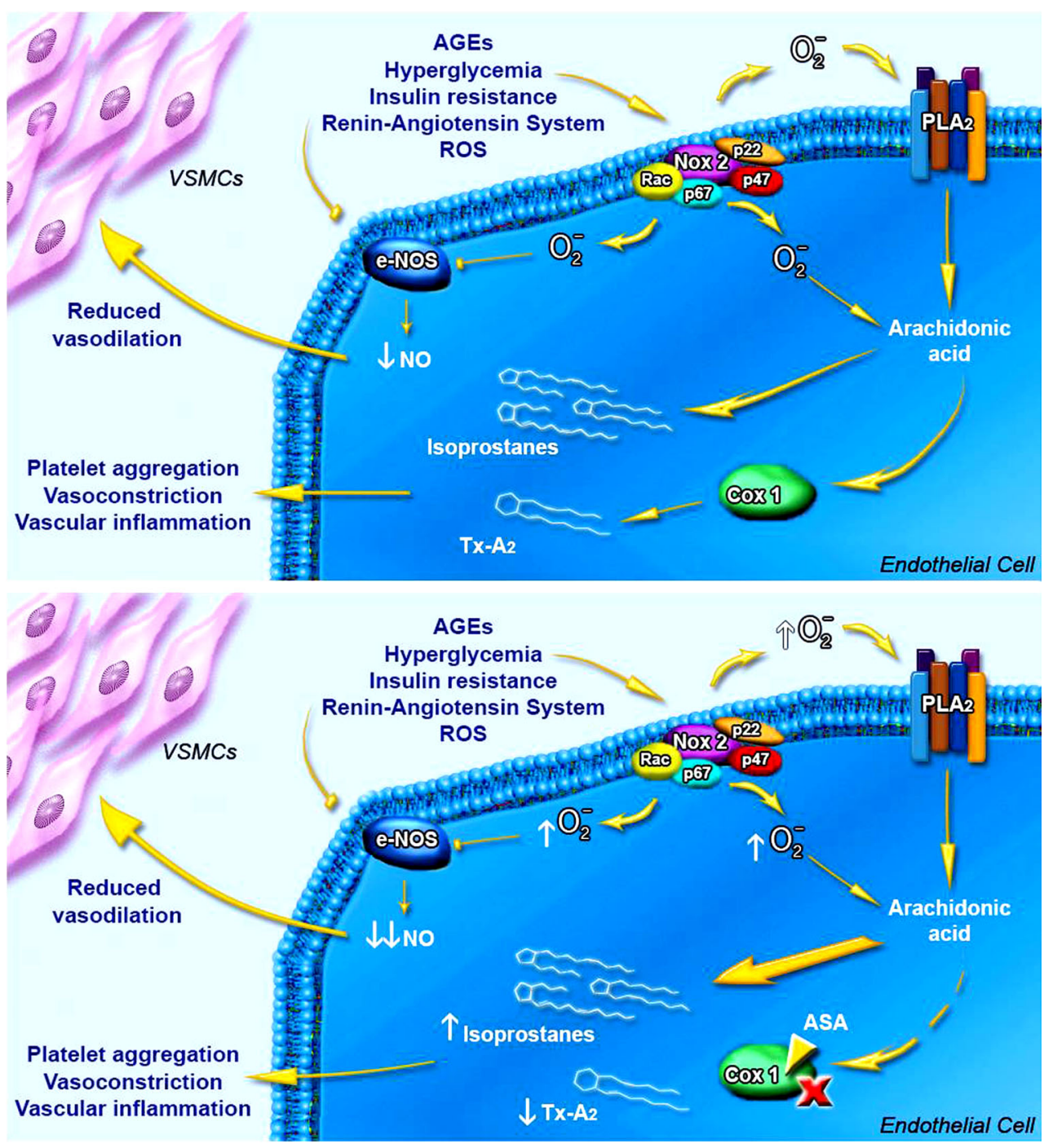

antidiabetic, and antihypertensive drugs, while our patients were newly diagnosed and without any pharmacological treatment. In addition, the Authors evaluated total antioxidant status and blood total glutathione, while we investigated the specific pathway involving NOX2 that is overexpressed in diabetic patients.

Thus, present data, if confirmed on a larger study population, could permanently discourage the use of the ASA for the primary prevention in patients with DM, providing also new targets for future research in the prevention and treatment of oxidative stress and endothelial dysfunction related to it.

\section{Study limitations}

The most relevant limitation of the study is the small number of patients, even if the statistical power size calculation is satisfied. This is due to the fact that the patients were enrolled at the first time of diagnosis of DM, after performing an OGTT. In addition, the aim of this study was to investigate a pathophysiological mechanism; thus, this study could be considered a pivotal one, and not an interventional one. The short duration of observation period (4 weeks) could be another limitation of the study. However, the demonstration that ASA induces a short-term negative effect, by the increase of oxidative stress and related endothelial dysfunction, could have relevant pathophysiological implications in the initial phases of vascular injury, such as in the restenosis after percutaneous coronary intervention.

Conflict of interest Eliezer Joseph Tassone, Maria Perticone, Angela Sciacqua, Simona Fortunata Mafrici, Chiara Settino, Natalia Malara, Vincenzo Mollace, Giorgio Sesti and Francesco Perticone declare that they have no conflict of interest.

Human and animal rights All procedures followed were in accordance with the ethical standards of the responsible committee on human experimentation (institutional and national) and with the Helsinki Declaration of 1975, as revised in 2008. 
Informed consent Informed consent was obtained from all patients for being included in the study.

Open Access This article is distributed under the terms of the Creative Commons Attribution License which permits any use, distribution, and reproduction in any medium, provided the original author(s) and the source are credited.

\section{References}

1. Beckman JA, Creager MA, Libby P (2002) Diabetes and atherosclerosis: epidemiology, pathophysiology, and management. JAMA 287:2570-2581

2. Haffner SM, Lehto S, Rönnemaa T, Pyörälä K, Laakso M (1998) Mortality from coronary heart disease in subjects with type 2 diabetes and in non diabetic subjects with and without prior myocardial infarction. N Engl J Med 339:229-234

3. Perticone F, Maio R, Sciacqua A, Andreozzi F, Iemma G, Perticone M, Zoccali C, Sesti G (2008) Endothelial dysfunction and C-reactive protein are risk factors for diabetes in essential hypertension. Diabetes 57:167-171

4. Presta I, Tassone EJ, Andreozzi F, Perticone M, Sciacqua A, Laino I, Musca D, Martino F, Sesti G, Perticone F (2011) Angiotensin II type 1 receptor, but no type 2 receptor, interferes with the insulin-induced nitric oxide production in HUVECs. Atherosclerosis 219:463-467

5. Newsholme P, Haber EP, Hirabara SM, Rebelato EL, Procopio J, Morgan D, Oliveira-Emilio HC, Carpinelli AR, Curi R (2007) Diabetes associated cell stress and dysfunction: role of mitochondrial and non-mitochondrial ROS production and activity. J Physiol 583:9-24

6. Basili S, Raparelli V, Riggio O, Merli M, Carnevale R, Angelico F, Tellan G, Pignatelli P, Violi F, CALC Group (2011) NADPH oxidase-mediated platelet isoprostane over-production in cirrhotic patients: implication for platelet activation. Liver Int 31:1533-1540

7. Pignatelli P, Carnevale R, Pastori D, Cangemi R, Napoleone L, Bartimoccia S, Nocella C, Basili S, Violi F (2012) Immediate antioxidant and antiplatelet effect of atorvastatin via inhibition of Nox2. Circulation 126:92-103

8. Carnevale R, Iuliano L, Nocella $\mathrm{C}$, Bartimoccia $\mathrm{S}$, Trapè $\mathrm{S}$, Russo R, Gentile MC, Cangemi R, Loffredo L, Pignatelli P, Violi F, IPINET group (2013) Relationship between platelet and urinary 8-Iso-PGF2 $\alpha$ levels in subjects with different degrees of NOX2 regulation. J Am Heart Assoc 2:e00198

9. FitzGerald GA (1991) Mechanisms of platelet activation: thromboxane A2 as an amplifying signal for other agonists. Am J Cardiol 68:11-15

10. Antithrombotic Trialists' Collaboration (2002) Collaborative meta-analysis of randomised trials of antiplatelet therapy for prevention of death, myocardial infarction, and stroke in high risk patients. BMJ 324:71-86

11. Ferroni P, Basili S, Falco A, Davì G (2004) Platelet activation in type 2 diabetes mellitus. J Thromb Haemost 2:1282-1291

12. Berger JS, Roncaglioni MC, Avanzini F, Pangrazzi I, Tognoni G, Brown DL (2006) Aspirin for the primary prevention of cardiovascular events in women and men: a sex-specific meta-analysis of randomized controlled trials. JAMA 295:306-313
13. Butalia S, Leung AA, Ghali WA, Rabi DM (2011) Aspirin effect on the incidence of major adverse cardiovascular events in patients with diabetes mellitus: a systematic review and metaanalysis. Cardiovasc Diabetol 10:25

14. American Diabetes Association (2013) Standards of medical care in diabetes-2013. Diabetes Care 36(Suppl 1):S11-S66. doi:10. 2337/dc13-S011

15. De Berardis G, Sacco M, Strippoli GF, Pellegrini F, Graziano G, Tognoni G, Nicolucci A (2009) Aspirin for primary prevention of cardiovascular events in people with diabetes: meta-analysis of randomised controlled trials. BMJ 339:b4531

16. Cangemi R, Pignatelli P, Carnevale R, Nigro C, Proietti M, Angelico F, Lauro D, Basili S, Violi F (2012) Platelet isoprostane overproduction in diabetic patients treated with aspirin. Diabetes 61:1626-1632

17. American Diabetes Association (2011) Diagnosis and classification of diabetes mellitus (2011). Diabetes Care 34(Suppl 1):S62S69. doi:10.2337/dc11-S062

18. Sakariassen KS, Femia EA, Daray FM, Podda GM, Razzari C, Pugliano M, Errasti AE, Armesto AR, Nowak W, Alberts P, Meyer JP, Sorensen AS, Cattaneo M, Rothlin RP (2012) EV-077 in vitro inhibits platelet aggregation in type-2 diabetics on aspirin. Thromb Res 130:746-752

19. Santilli F, Mucci L, Davì G (2011) TP receptor activation and inhibition in atherothrombosis: the paradigm of diabetes mellitus. Intern Emerg Med 6:203-212

20. Pignatelli P, Carnevale R, Di Santo S, Bartimoccia S, Sanguigni V, Lenti L, Finocchi A, Mendolicchio L, Soresina AR, Plebani A, Violi F (2011) Inherited human gp91phox deficiency is associated with impaired isoprostane formation and platelet dysfunction. Arterioscler Thromb Vasc Biol 31:423-434

21. Capra V, Bäck M, Angiolillo DJ, Cattaneo M, Sakariassen KS (2013) Impact of vascular TP receptor activation on hemostasis, thrombosis, oxidative stress and inflammation. J Thromb Haemost 12:126-137

22. Gonçalves LH, Silva MVF, Duarte RCF, Dusse LMS, Fernandes AP, Bosco AA, Gomes KB, Carvalho MG (2014) Acetylsalicylic acid therapy: influence of metformin use and other variables on urinary 11-dehydrothromboxane $\mathrm{B}_{2}$ levels. Clin Chim Acta 429:76-78

23. Perticone F, Ceravolo R, Pujia A, Ventura G, Iacopino S, Scozzafava A, Ferraro A, Chello M, Mastroroberto P, Verdecchia P, Schillaci G (2001) Prognostic significance of endothelial dysfunction in hypertensive patients. Circulation 104:191-196

24. Perticone F, Ceravolo R, Candigliota M, Ventura G, Iacopino S, Sinopoli F, Mattioli PL (2001) Obesity and body fat distribution induce endothelial dysfunction by oxidative stress: protective effect of vitamin C. Diabetes 50:159-165

25. Ogawa H, Nakayama M, Morimoto T, Uemura S, Kanauchi M, Doi N, Jinnouchi H, Sugiyama S, Saito Y, Japanese Primary Prevention of Atherosclerosis with Aspirin for Diabetes (JPAD) Trial Investigators (2008) Low-dose aspirin for primary prevention of atherosclerotic events in patients with type 2 diabetes: a randomized controlled trial. JAMA 300:2134-2141

26. Raghavan RP, Laight DW, Cummings MH (2014) Aspirin in type 2 diabetes, a randomised controlled study: effect of different doses on inflammation, oxidative stress, insulin resistance and endothelial function. Int J Clin Pract 68:271-277 